\title{
Tuberculose: a calamidade negligenciada
}

\author{
Tuberculosis: the negleted calamity
}

\author{
Antonio Ruffino-Netto ${ }^{1}$
}

Resumo Neste artigo, o autor atualiza dados epidemiológicos sobre a tuberculose e descreve a luta contra a doença no Brasil e no mundo. Destaca aspectos históricos sobre as políticas do controle da doença no país e examina o Plano Nacional de Controle da Tuberculose ora vigente. Termina assinalando alguns problemas pendentes e discute novas alternativas para o controle da tuberculose.

Palavras-chaves: Tuberculose. Epidemiologia da tuberculose. Programa de controle da tuberculose.

Abstract Epidemiological data on tuberculosis and the fight against the disease in Brazil and in the world are reviewed herein. Historical aspects of the different approaches to the control of the disease in Brazil are highlighted and the ongoing Brazilian Program for the Control of Tuberculosis is examined. To conclude, the author takes a look at unresolved problems and considers new alternatives for the control of tuberculosis.

Key-words: Tuberculosis. Epidemiology of tuberculosis. Control of tuberculosis.

A tuberculose $(\mathrm{Tb})$ tem preocupado as autoridades da área da saúde em todo o mundo. Em 1993, a Organização Mundial da Saúde (OMS) declarou a situação da tuberculose como estado de urgência. Esta própria instituição se deu conta que, sozinha, não conseguiria controlar a doença. Criou-se, então, o programa "STOP TB" que reune instituições de alto nível científico e/ou poder econômico, tais como: a Organização Mundial da Saúde, o Banco Mundial, o Centers for Disease Control (CDC)Atlanta, International Union Against Tuberculosis and Lung Disease (IUATLD), Royal Netherlands Tuberculosis Association (RNTA) e American Thoracic Association (ATA).

AOMS ${ }^{11}$ assinala como principais causas para a gravidade da situação atual da tuberculose no mundo os seguintes fatos: desigualdade social, advento da AIDS, envelhecimento da população, grandes movimentos migratórios.

Deve-se assinalar que, em muitos locais (assim como acreditam muitos profissionais de saúde), as autoridades deixaram o problema de lado como se fosse assunto resolvido. Afinal, já se conhecia bastante a enfermidade, sua fisiopatologia, diagnóstico, esquemas terapêuticos e medicamentos disponíveis. Não se considerou que a existência de todo esse saber prévio ou recursos de nada adiantam se eles não forem colocados ao alcance da população geral e, acima de tudo, que esta população necessita fazer uso efetivo dos recursos quando estes existem no local.

Os erros de avaliação do problema resultaram em medidas tímidas no sentido de implementar e por em funcionamento todos os recursos disponíveis, sejam os conhecimentos, sejam os serviços. Julgamos isso um descalabro consentido que abarcou desde políticas públicas do Ministério da Saúde (MS), das Secretarias Estaduais (SES), e Municipais de Saúde (SMS) e aparelhos formadores dos profissionais de saúde, ou seja, as universidades.

Tem-se chamado a atenção ${ }^{6}$ sobre as doenças emergentes (e.g., AIDS) ou reemergentes (dengue, por exemplo). Alguns chegam a alegar ser a tuberculose um problema reemergente em nosso meio. Essa afirmativa poderá ser válida para alguns paises europeus, e mesmo para os Estados Unidos da América, contudo, não é valida para o Brasil: para nós, a tuberculose não é problema de saúde pública emergente e tampouco reemergente. Ela é um problema presente e ficante há longo tempo.

\footnotetext{
1. Faculdade de Medicina de Ribeirão Preto da Universidade de São Paulo, Ribeirão Preto, SP.

Endereço para correspondência: Dr. Antonio Ruffino-Netto. Depto de Medicina Social/FMRP/USP. Av. Bandeirantes 3900, 14049-900 Ribeirão Preto, SP, Brasil Tel: 5516 602-3070; Fax: 5516 633-1386

e-mail: aruffino@fmrp.usp.br

Recebido para publicação em 24/09/2001.
} 
Sabe-se que cerca de $95 \%$ dos casos de Tb ocorrem no terceiro mundo, e, aí ocorrem $98 \%$ dos óbitos ${ }^{11}$. Em 22 países concentram-se $80 \%$ dos casos estimados para o mundo, entre os quais encontra-se o Brasil ocupando o 10\% lugar (em 1997). Em publicação da $\mathrm{OMS}^{12}$, de 1998, o Brasil passou a ocupar o $13^{\circ}$ lugar.
Apresentam-se nas Tabelas 1 e 2, respectivamente, a distribuição dos casos notificados e os coeficientes de incidência de Tb no Brasil em 1999, segundo a Unidade da Federação e segundo o tipo de doença. Observa-se que o maior número de casos notificados ocorreu em ordem decrescente nos Estados de São

Tabela 1 - Tuberculose: distribuição de casos ntificados no Brasil e unidades federadas, 1999.

\begin{tabular}{|c|c|c|c|c|c|}
\hline \multirow{2}{*}{$\begin{array}{l}\text { Unidades Federadas/ } \\
\text { Macro Região }\end{array}$} & \multicolumn{2}{|c|}{ Pulmonar } & \multirow{2}{*}{ Extrapulmonar } & \multirow{2}{*}{ Todas as formas } & \multirow{2}{*}{ População } \\
\hline & Bacilifera & Total & & & \\
\hline Brasil & 41.434 & 66.307 & 12.153 & 78.628 & 163.947 .554 \\
\hline Norte & 4.216 & 5.668 & 812 & 6.480 & 12.133 .705 \\
\hline Rondônia & 331 & 499 & 59 & 558 & 1.296 .856 \\
\hline Acre & 238 & 354 & 23 & 377 & 527.937 \\
\hline Amazonas & 1.288 & 1.783 & 351 & 2.134 & 2.580 .860 \\
\hline Roraima & 103 & 175 & 24 & 199 & 266.922 \\
\hline Amapá & 134 & 178 & 30 & 208 & 439.781 \\
\hline Tocantins & 165 & 256 & 20 & 276 & 1.134 .895 \\
\hline Nordeste & 12.295 & 20.644 & 2.544 & 23.356 & 46.289 .042 \\
\hline Maranhão & 1.361 & 2.613 & 148 & 2.929 & 5.418 .349 \\
\hline Piauí & 719 & 1.111 & 212 & 1.323 & 2.734 .152 \\
\hline Ceará & 2.229 & 3.245 & 333 & 3.578 & 7.106 .605 \\
\hline Rio G. do Norte & 570 & 993 & 122 & 1.115 & 2.654 .501 \\
\hline Paraíba & 667 & 1.174 & 148 & 1.322 & 3.375 .609 \\
\hline Alagoas & 627 & 942 & 156 & 1.098 & 2.713 .203 \\
\hline Sergipe & 340 & 520 & 76 & 596 & 1.712 .786 \\
\hline Bahia & 4.015 & 6.889 & 905 & 7.794 & 12.993 .011 \\
\hline Sudeste & 18.132 & 29.576 & 6.329 & 35.905 & 69.858 .115 \\
\hline Minas Gerais & 3.046 & 4.251 & 1.085 & 5.336 & 17.295 .955 \\
\hline Espírito Santo & 885 & 1.148 & 240 & 1.388 & 2.938 .062 \\
\hline Rio de Janeiro & 5.224 & 8.944 & 1.835 & 10.779 & 13.807 .358 \\
\hline São Paulo & 8.977 & 15.233 & 3.169 & 18.402 & 35.816 .740 \\
\hline Sul & 4.848 & 7.239 & 1.864 & 9.103 & 24.445 .950 \\
\hline Paraná & 1.384 & 2.261 & 501 & 2.762 & 9.375 .592 \\
\hline Santa Catarina & 770 & 1.201 & 324 & 1.525 & 5.098 .448 \\
\hline Rio G. do Sul & 2.694 & 3.777 & 1.039 & 4.816 & 9.971 .910 \\
\hline Centro-Oeste & 1.943 & 3.180 & 604 & 3.784 & 11.220 .742 \\
\hline Mato G. do Sul & 439 & 771 & 145 & 916 & 2.026 .600 \\
\hline Mato Grosso & 601 & 1.068 & 124 & 1.192 & 2.375 .549 \\
\hline Goiás & 615 & 846 & 188 & 1.034 & 4.848 .725 \\
\hline
\end{tabular}

Paulo, Rio de Janeiro, Bahia, Minas Gerais e Rio Grande do Sul. Em termos de coeficientes de notificação, esta ordem se altera para: Amazonas, Rio de Janeiro, Roraima e Acre.

O Programa Stop Tb promoveu a reunião dos 22 países em junho de 1999 em Casablanca, Marrocos, com o intuito de discutir pesquisas na área da tuberculose. Em julho de 1999, o mesmo Programa reuniu em Brasília, DF, 9 países da América do Sul (onde a prevalência da Tb é muito grande) para discutir a situação nas Américas. Em janeiro de 2000, o Programa reuniu os 22 países em Bangkok, na Thailandia, para se discutir a preparação da Conferência Ministerial sobre Tuberculose e Desenvolvimento Sustentável que foi efetuada em março de 2000 em Amsterdam, na Holanda. Em Bangkok, discutiu-se a situação dos Programas de Controle da Tuberculose (PCT) efetuados nos 22 países, considerando-se os pontos fracos, pontos fortes, oportunidades, desafios e perspectivas. Nessa época, ficou bastante claro que havia países onde os principais problemas do PCT estavam afeitos à falta de recursos financeiros e humanos; em outros países os problemas eram mais ligados a multidroga resistência (MDR) e/ou gerenciamento dos programas.

$\mathrm{Na}$ reunião de Amsterdam, foi publicada a Amsterdam Declaration on Stop $T b^{13}$ assinada pelos Ministros de Saúde dos 22 países participantes. Essa declaração foi assinada no dia 24 de março de 2000, quando se comemorava o Dia Mundial de Combate a Tuberculose. Na declaração, reitera-se o compromisso dos países envolvidos em priorizar a luta contra a doença bem como enfatiza-se a importância de buscar parceiros em organizações não-governamentais (ONGs) para a realização dessa tarefa. 
Tabela 2 - Tuberculose: coeficientes de incidência no Brasil e unidades federadas, 1999.

\begin{tabular}{|c|c|c|c|c|}
\hline \multirow{2}{*}{$\begin{array}{l}\text { Unidades Federadas/ } \\
\text { Macro Região }\end{array}$} & \multicolumn{4}{|c|}{ Coeficientes de incidência por 100.000 habitantes } \\
\hline & Bacilifera & Pulmonar & Extrapulmonar & Todas as formas \\
\hline Brasil & 25,3 & 40,4 & 7,4 & 48,0 \\
\hline Rondônia & 25,5 & 38,5 & 4,5 & 43,0 \\
\hline Acre & 45,1 & 67,1 & 4,4 & 71,4 \\
\hline Amazonas & 49,9 & 69,1 & 13,6 & 82,7 \\
\hline Roraima & 38,6 & 65,6 & 9,0 & 74,6 \\
\hline Pará & 33,2 & 41,2 & 5,2 & 46,3 \\
\hline Amapá & 30,5 & 40,5 & 6,8 & 47,3 \\
\hline Tocantins & 14,5 & 22,6 & 1,8 & 24,3 \\
\hline Nordeste & 26,6 & 44,6 & 5,5 & 50,5 \\
\hline Maranhão & 25,1 & 48,2 & 2,7 & 54,1 \\
\hline Piauí & 26,3 & 40,6 & 7,8 & 48,4 \\
\hline Ceará & 31,4 & 45,7 & 4,7 & 50,3 \\
\hline Rio G. do Norte & 21,5 & 37,4 & 4,6 & 42,0 \\
\hline Alagoas & 23,1 & 34,7 & 5,7 & 40,5 \\
\hline Sergipe & 19,9 & 30,4 & 4,4 & 34,8 \\
\hline Bahia & 30,9 & 53,0 & 7,0 & 60,0 \\
\hline Sudeste & 26,0 & 42,3 & 9,1 & 51,4 \\
\hline Minas Gerais & 17,6 & 24,6 & 6,3 & 30,9 \\
\hline Espírito Santo & 30,1 & 39,1 & 8,2 & 47,2 \\
\hline Rio de Janeiro & 37,8 & 64,8 & 13,3 & 78,1 \\
\hline São Paulo & 25,1 & 42,5 & 8,8 & 51,4 \\
\hline Sul & 19,8 & 29,6 & 7,6 & 37,2 \\
\hline Paraná & 14,8 & 24,1 & 5,3 & 29,5 \\
\hline Santa Catarina & 15,1 & 23,6 & 6,4 & 29,9 \\
\hline Rio G. do Sul & 27,0 & 37,9 & 10,4 & 48,3 \\
\hline Centro-Oeste & 17,3 & 28,3 & 5,4 & 33,7 \\
\hline Mato G. do Sul & 21,7 & 38,0 & 7,2 & 45,2 \\
\hline Mato Grosso & 25,3 & 45,0 & 5,2 & 50,2 \\
\hline Goiás & 12,7 & 17,4 & 3,9 & 21,3 \\
\hline
\end{tabular}

\section{POLÍTICAS DE CONTROLE DA TUBERCULOSE NO BRASIL}

A tuberculose seguramente foi introduzida no Brasil pelos portugueses e missionários jesuítas, a partir do ano de $1500^{7}$.

Ruffino-Netto e Pereira ${ }^{8}$ apresentam um estudo da evolução da mortalidade por Tb no Rio de Janeiro, no período de 1860 a 1960 , onde analisam os fatores econômicos, demográficos, sociais e as políticas de saúde aí existentes, desde a chegada da Família Real aqui no Brasil em 1808 até o final da década de 80. Discutem o fenômeno da reeuropeização da cidade do Rio de Janeiro. Dado o fastígio da cultura do café, que antecedeu o período de 1885, houve grandes transformações na sociedade de então. Em termos de políticas de saúde, deve-se assinalar que o Dr. Paula Candido, presidindo a Junta Central de Higiene da capital do império, conseguiu junto ao Parlamento que fossem adotadas novas medidas sanitárias para o início do controle da $\mathrm{Tb}$. O Decreto $\mathrm{n}^{\circ} 6.387$, de 15 de novembro de 1876, reorganiza os serviços sanitários nas cidades do império. A partir de 1870 , foram baixadas várias leis relacionadas com a habitação. De 1876 a 1886, baixaram-se cinco decretos e um aviso ministerial relativos à polícia sanitária domiciliar.
No início do século 19 a assistência ocorria através de organizações filantrópicas, quando se observava que um terço dos óbitos em geral deviam-se à tuberculose. No início do século 20, Oswaldo Cruz reconhece a necessidade da atenção das autoridades sanitárias sobre a Tb; ele procurou instituir um plano de ação para o combate da enfermidade, alcançando, contudo, pequeno impacto. Com a Reforma Carlos Chagas, no final da década de 10, criou-se a Inspetoria de Profilaxia da Tuberculose. Esta preconizava o diagnóstico e tratamento dos casos de Tb.

Em 1927, Arlindo de Assis aplica pela primeira vez o BCG oral em recém-nascidos. Em 1940, o Ministro Gustavo Capanema criou o Plano Federal de Construção de Sanatórios que previa o término do Sanatório do Distrito Federal, que fora iniciado em 1937.

Em 1941, foi criado o Serviço Nacional de Tuberculose (SNT) com a função de estudar os problemas relativos a tuberculose e ao desenvolvimento de meios de ação profilática e assistencial. Em 1946, instalou-se a Campanha Nacional Contra a Tuberculose (CNCT) tendo por objetivos: coordenar todas as atividades de controle da doença, uniformizar a 
orientação nacional, sugerir descentralização dos serviços e efetuar cadastramento torácico da população. O seu impacto foi imediato no Programa de Controle da Tuberculose.

A partir da década de 40 , começa também grande alteração na tendência da mortalidade por Tb em decorrência da utilização dos tuberculostáticos: estreptomicina (SM) a partir de 1948; ácido paraamino-salicílico (PAS) a partir de 1949; hidrazida (INH) a partir de 1952.

A partir da década de 60 , começa efetivamente a utilização de esquemas terapêuticos padronizados. Em 1964, utilizava-se o esquema padrão de 18 meses de duração (SM+INH+PAS). Em 1965, o esquema terapêutico é reduzido para 12 meses.

Em 1970, instala-se a Divisão Nacional de Tuberculose (DNT) substituindo o antigo SNT. Em 1971, cria-se a Central de Medicamentos (CEME) com o objetivo de fornecer tuberculostáticos para todos os doentes com Tb do país.

Em 1973, implanta-se a vacinação com BCG intradérmica, sendo obrigatória a vacinação para menores de um ano de idade a partir de 1976.

Em 1975, inaugura-se o II Plano Nacional de Desenvolvimento, que inclui o Programa de Controle da Tuberculose financiado pelo MS/INAMPS/SES, tendo por objetivo integrar os diferentes níveis do governo para reduzir a morbidade, mortalidade e problemas sócioeconômicos decorrentes da Tb. Em 1976, organiza-se a Divisão Nacional de Pneumologia Sanitária (DNPS) cuja abrangência era um tanto indefinida no momento da criação. Houve assim diminuição dos poderes e autonomia do antigo DNT.

O esquema de tratamento de curta duração (6 meses) utilizando a Rifampicina (RMP) foi introduzido em 1979 (RMP+INH+PZA).

Em 1981, é assinado o convênio entre INAMPS/ SES/MS objetivando transferir a execução do controle da Tb para as SES. A partir de 1981 aparecem novas estratégias de organização dos serviços de saúde: Ações Integradas de Saúde (AIS), Sistema Único e Descentralizado de Saúde (SUDS) e finalmente o Sistema Único de Saúde (SUS).

Embora a OMS apresente o tratamento supervisionado como uma proposta inovadora, devese ressaltar contudo que a Fundação de Serviço Especial de Saúde Pública (SESP), do Brasil, já utilizava o tratamento segundo esta estratégia a partir de 1962, em unidades de todos os níveis de complexidade. $\mathrm{O}$ tratamento supervisionado era feito pela enfermagem. Em 1981, o programa conseguiu cobrir todas as unidades da Fundação ${ }^{5}$.

Em 1990, criou-se a Coordenação Nacional de Pneumologia Sanitária (CNPS), ligada à Fundação Nacional de Saúde (FUNASA) e é extinta a CNCT pelo Presidente Fernando Collor de Mello. Há desestruturação do PCT em nível federal, não priorização do programa de controle, enfraquecimento das coordenações estaduais, diminuição dos recursos financeiros, diminuição das supervisões do programa, disseminação da AIDS, queda da cobertura, diminuição da busca de casos novos, piora dos resultados de tratamento, aumento do abandono. Veja análise detalhada sobre o controle da doença no período de 20 anos (de 1978 a 1998) em publicação do Boletim de Pneumologia Sanitária?.

Em 1992, na tentativa de reerguer o PCT, há a transferência da responsabilidade dos treinamentos, monitorização dos tratamentos e campanhas públicas para os Estados.

Dado que, em 1993, a OMS declarou o estado de urgência da tuberculose, o MS elaborou o Plano Emergencial para o controle da enfermidade que, efetivamente, foi implantado a partir de 1996. Tinha como objetivo aumentar a efetividade das ações de controle através da implementação de atividades específicas em 230 municípios prioritários onde se concentrava $75 \%$ dos casos estimados para o Brasil, visando diminuir a transmissão do bacilo na população até o ano 1998. A escolha destes municípios prioritários baseou-se em critérios de magnitude epidemiológica da Tb e da AIDS, tamanho da população, bem como informações operacionais. O plano foi elaborado e fundamentado através de repasse de recursos financeiros aos municípios, à base de $\mathrm{R} \$ 100,00$ (cem reais) para cada caso estimado de tuberculose que deveria ser descoberto no referido município. O repasse era operacionalizado através de convênio entre o município e a FUNASA. O convênio estipulava obrigações/restrições na aplicação dos recursos (Ministério da Saúde, 1996) ${ }^{2}$

Como veremos adiante, foi um plano montado sob a óptica de uma administração burocrática.

Em março de 1998, a imprensa internacional chama a atenção para a calamidade da situação epidemiológica da tuberculose no mundo. O Conselho Nacional de Saúde, em 6 de agosto de 1998, estabelece a resolução número 284 , considera a situação da doença no país, e conclui que o Plano Emergencial ainda em implementação requeria ajustes e ampliação, e que a situação poderia e deveria ser corrigida com recursos disponíveis; resolve, por fim, que a tuberculose era problema prioritário de saúde pública no Brasil (tanto por sua magnitude como pela possibilidade e vantagens do seu controle) e sugere estabelecer estratégias para um novo plano.

Neste mesmo ano, a CNPS volta ao controle do Ministério da Saúde, vinculada à Secretaria de Políticas de Saúde (SPS).

Em outubro de 1998, lançou-se o Plano Nacional de Controle da Tuberculose ${ }^{3}$, com as seguintes diretrizes gerais: 1) o MS é responsável pelo estabelecimento das normas; 2) a aquisição e abastecimento de medicamentos; 3 ) referência laboratorial e de tratamento; 4) coordenação do sistema de informações; 
5) apoio aos Estados e Municípios; 6) articulação intersetorial visando maximizar os resultados de políticas públicas.

Reconhece que a condição essencial é a articulação e a complementaridade de ações dos três níveis de gestão do SUS (União, Estados e Municípios); envolver obrigatoriamente a participação social e organizações não governamentais. Deteç̧ão e diagnóstico feitos fundamentalmente através da baciloscopia em todos os sintomáticos respiratórios (SR) e contatos. Disponibilizar tuberculostáticos, incluindo um estoque estratégico, assegurar tratamento supervisionado e vigilância da resistência das drogas. Prover um sistema de informações de acordo com recomendações da OMS.

O Plano introduz duas inovações: o tratamento supervisionado e a instituição de um bônus de $R$ \$150,00 e de $R \$ 100,00$ para cada caso de doente de tuberculose diagnosticado, tratado e curado, respectivamente, se foi utilizado ou não o tratamento supervisionado. $O$ repasse desse bônus é feito automaticamente por ocasião da notificação da alta curado do paciente. Fica suprimida toda e qualquer burocracia de assinaturas de convênio para esses repasses. Observa-se, então, uma substituição da administração burocrática por uma administração gerencial.

\section{A NOVA GERÊNCIA PROPOSTA}

Este último plano tem contemplado os $5 \mathrm{Rs}$ : reestruturação, reengenharia, reinvenção, realinhamento e reconceitualização, no gerenciamento público, proposto por Jones e Thompson'.

Reestruturação. O plano propõe utilizar melhor a escassa estrutura existente, eliminando tudo que não agregue valor aos serviços, isto é, foram eliminados os convênios. Esforço foi feito no sentido dos serviços de saúde repensarem seus resultados, levando-se em consideração o que apresentavam de estrutura, como estava elaborado o processo de atendimento (o esquema na Figura 1 ajuda a refletir sobre a composição do sistema de saúde e buscar maneiras de aumentar sua eficácia/eficiência).

\section{ELEMENTOS QUE COMPÕEM O SISTEMA DE ATENÇÃO À SAÚDE}

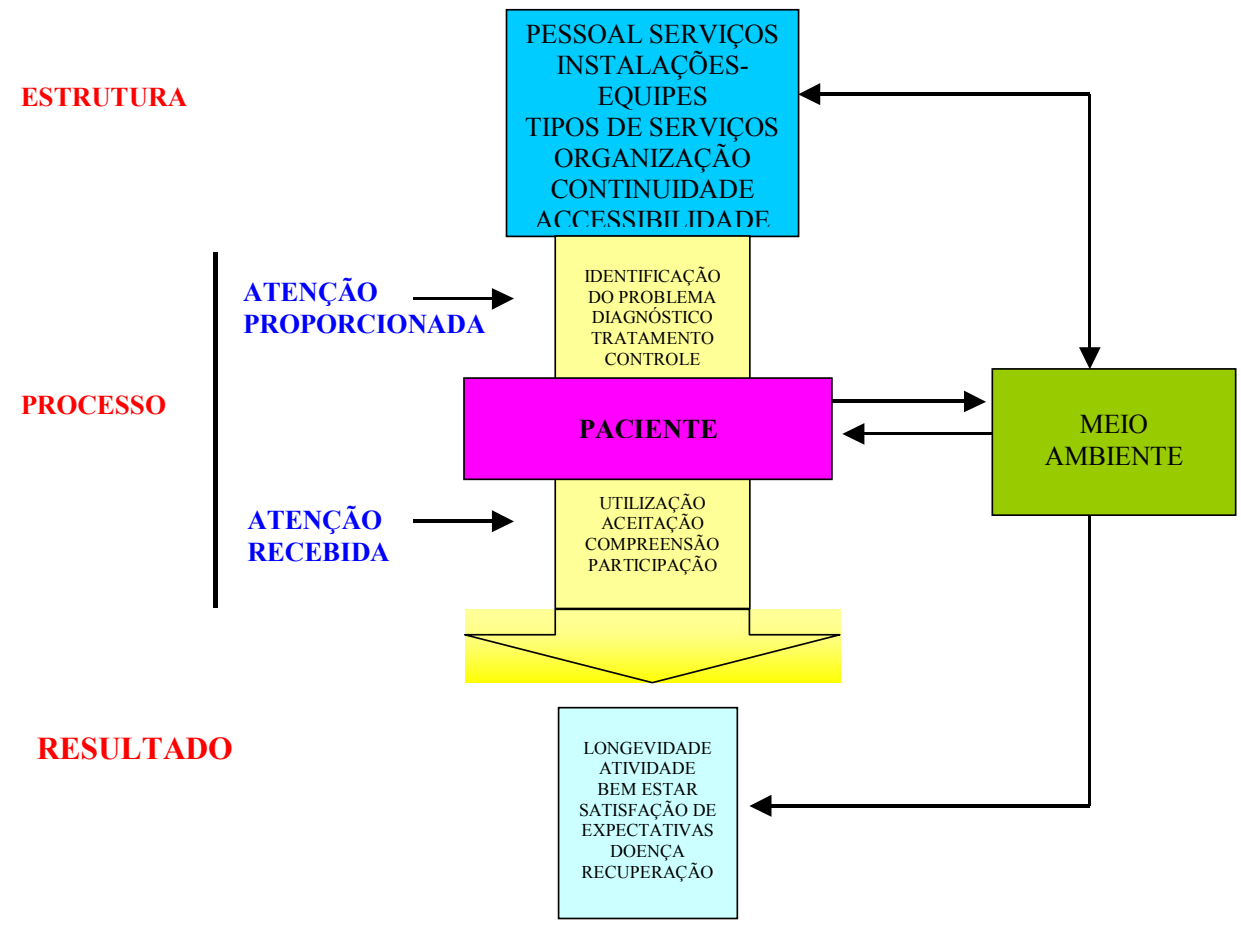

Figura 1 - Os elementos que compõem o sistema de atenção à saúde. Modificado de Starfield (1992). 
Reengenharia. O plano foi concebido com novas bases e utilizando nova lógica de repasse de recursos financeiros para os municípios. Esta nova lógica altera radicalmente a forma de focalizar e pensar o plano de atividades. Para se obter recursos financeiros, o serviço de saúde tem que mostrar resultados, isto é, curar pacientes de tuberculose e devolver estes pacientes efetivamente curados para a sociedade. Isto significa pensar na forma de procurar os suspeitos, identificá-los, diagnosticar a doença, tratar e obter boa adesão ao tratamento. Deve-se acrescentar que caberá ao município/estado empenho em melhorar o seu sistema de informações para notificar casos e notificar as curas. No início da implantação de tal sistemática, alguns estados da federação encontraram dificuldade em sua operacionalização. Há exemplos importantes em que apenas com alterações no processo e não propriamente nas estruturas dos serviços alguns conseguiram resultados surpreendentes na adesão ao tratamento. A Paraíba representa um bom exemplo; o estado reduziu o percentual de abandono do tratamento de $12 \%$ para $1,9 \%$. Em nível local, o exemplo mais marcante foi Cuiabá, onde o percentual de abandono caiu de $50 \%$ para $4 \%$.

Reinvenção. A OMS vem apregoando desde 1993 (WHO, 1999), a estratégia Directly Observed Treatment Short-course (DOTS) que envolve 5 componentes: comprometimento político com o programa, detecção de casos pela baciloscopia, tratamento de curta duração e diretamente observado, regularidade na manutenção de medicamentos e sistema de informações que permita um monitoramento dos resultados. Observa-se que o tratamento supervisionado é um dos componentes que foi oficialmente introduzido com o plano referido. No início, havia pelo menos uma unidade de demonstração para cada estado da federação. No ano de 1999 existiam apenas cerca de 100 unidades sanitárias no país utilizando o tratamento supervisionado. No final do ano 2000, já passaram a existir 1.106 unidades (Tabela 3). Outro componente da estratégia DOTS é a feitura do

Tabela 3 - Distribuição das unidades sanitárias com tratamento supervisionado segundo as unidades da federação - Brasil, $P C T=$ Programa de Controle da Tuberculose .

\begin{tabular}{|c|c|c|c|c|c|}
\hline \multirow[b]{2}{*}{$\begin{array}{l}\text { Unidades } \\
\text { Federadas }\end{array}$} & \multirow{2}{*}{$\begin{array}{l}\text { Total de unid. sanitárias } \\
\text { integradas ao PCT }\end{array}$} & \multicolumn{4}{|c|}{ Número de unidades com tratamento supervisionado } \\
\hline & & $\begin{array}{l}\text { Já implantado início } \\
2000\end{array}$ & $\begin{array}{l}\text { Previsão de implantação até } \\
\text { o final de } 2000\end{array}$ & $\begin{array}{l}N^{\circ} \text { efetivo } \\
\text { implantado final } \\
2000\end{array}$ & $\begin{array}{c}\% \text { cobertura das } \\
\text { metas }\end{array}$ \\
\hline Rondônia & 46 & 0 & 6 & 6 & 100,0 \\
\hline Acre & 35 & 0 & 0 & 0 & \\
\hline Amazonas & 99 & 1 & 8 & 1 & 12,5 \\
\hline Roraima & 28 & 9 & 0 & 9 & \\
\hline Pará & 586 & 0 & 0 & 13 & \\
\hline Amapá & 35 & 1 & 5 & 3 & 60,0 \\
\hline Tocantins & 214 & 31 & 27 & 72 & 266,0 \\
\hline Maranhão & 156 & 2 & 15 & 8 & 53,0 \\
\hline Piauí & 144 & 16 & 21 & 26 & 123,0 \\
\hline Ceará & 403 & 22 & 38 & 67 & 176,0 \\
\hline Rio G. do Norte & 306 & 208 & NR & 82 & \\
\hline Paraíba & 152 & 11 & 37 & 28 & 75,0 \\
\hline Pernambuco & 193 & 24 & 26 & 30 & 115,0 \\
\hline Alagoas & 158 & 5 & 7 & 67 & 957,0 \\
\hline Sergipe & 73 & 22 & 62 & 72 & 116,0 \\
\hline Bahia & 347 & 3 & NR & 13 & \\
\hline Minas Gerais & - & 0 & - & 0 & \\
\hline Espírito Santo & - & 10 & 22 & 45 & 204,0 \\
\hline Rio de Janeiro & - & 0 & NR & 5 & \\
\hline São Paulo & 1.180 & 219 & NR & 219 & \\
\hline Paraná & 751 & 0 & NR & 172 & \\
\hline Santa Catarina & 225 & 7 & 7 & 7 & 100,0 \\
\hline Rio G. do Sul & 323 & 1 & 17 & 5 & 29,0 \\
\hline Mato G. do Sul & 119 & 11 & 10 & 0 & 0,0 \\
\hline Mato Grosso & 177 & 77 & 40 & 139 & 347,0 \\
\hline Goiás & 193 & 5 & 7 & 15 & 214,0 \\
\hline Distrito Federal & 43 & 2 & 2 & 2 & 100,0 \\
\hline Total & 5.986 & 687 & 357 & 1.106 & 309,0 \\
\hline
\end{tabular}

diagnóstico pela baciloscopia, dado seu baixo custo. $\mathrm{O}$ plano apresentado propôs aumentar o preço da baciloscopia para aumentar sua utilização. Para se ter uma idéia da utilização deste recurso, basta assinalar que no Brasil, em 1997, para uma população de 160 milhões de habitantes, realizaram-se cerca de 300.000 baciloscopias diagnósticas. Se fôssemos fazer 2 baciloscopias para diagnóstico para cada SR (e supondo $1 \%$ da população geral nesta categoria) teríamos estimados 1.600.000 SR e portanto, 3.200.000 baciloscopias diagnósticas. Isto é, na época, antecedendo o plano, efetuava-se apenas $19 \%$ das 
baciloscopias diagnósticas esperadas. A título de comparação, lembramos que o Peru, para uma população de 23 milhões de habitantes, faz 1.400 .000 baciloscopias diagnósticas.

Realinhamento e reconceitualização. A partir de janeiro de 2000, a Área Técnica de Pneumologia Sanitária, foi incorporada ao Departamento de Atenção
Básica (DAB) da Secretaria de Políticas de Saúde do Ministério. O apoio diagnóstico e para tratamento oferecido pela estrutura do Programa de Saúde da Família (PSF) e dos Agentes Comunitários (PACS) poderá auxiliar bastante no tratamento supervisionado, bem como no sistema de registros e monitoramento dos casos.

\section{PROBLEMAS PENDENTES E PERSPECTIVAS}

Há problemas sérios na área da saúde pública. $O$ primeiro deles, é a continuidade dos programas. O segundo, a adequação das propostas. Às vezes, as propostas focalizam o problema da tuberculose como se ele representasse apenas um somatório dos tuberculosos existentes, mas na realidade, o problema inclui fundamentalmente a área social. Para o tuberculoso, a solução implica o uso de esquemas terapêuticos eficazes (tipo RMP+ INH+ PZA). Para a tuberculose, a solução exige programas que visem uma qualidade de vida mais adequada.

No Brasil, atualmente, ainda é pequeno o diálogo entre as universidades e os serviços de saúde pública no tema pertinente a tuberculose. No passado, este diálogo parece que já foi bem maior. Caberia à universidade colaborar com orientação metodológica e apontar novos caminhos para enfrentar o problema, novas técnicas diagnósticas (mais rápidas), ampliar estudos epidemiológicos utilizando biologia molecular para melhor entender a dinâmica da doença na comunidade.

Novos desafios despontam no novo século: diagnósticos mais rápidos, MDR, estudos sobre custo/ benefício, adesão ao tratamento, novas vacinas, novas estratégias pós-genomas, novos modelos explicativos para a doença.

Cabe aos serviços públicos (Ministério da Saúde, Secretarias de Saúde) também apoiar universidades, com recursos financeiros para as pesquisas operacionais.

Atualmente, está sendo implantado/implementado uma Rede de Pesquisas em Tuberculose no Brasil. Tal rede revela-se algo inédito. Ela se propõe a identificar todos as pessoas envolvidas em pesquisa em tuberculose, indo desde a área básica, que envolve pesquisas de ponta com biologia molecular, passando pelos kits diagnósticos, ensaios clínicos e chegando à comunidade com estudos epidemiológicos e operacionais, incluindo a interface destes estudos e a produção de medicamentos. Já existem centenas de pessoas cadastradas na rede.

$\mathrm{Pio}^{4}$ (ex-coordenador da OPAS para os PCT das Américas) comentando sobre os problemas e perspectivas da luta contra a tuberculose, levantava as seguintes questões em 1985: é realista a estratégia mundial de saúde para todos no ano 2000 , no que se refere ao controle da tuberculose? O que teremos alcançado no ano 2000? Assinala que, no final da década de 40, a disponibilidade de vacinação com BCG, radiografia de tórax em massa e quimioterapia específica criou um clima de grande otimismo com respeito ao futuro da tuberculose. A eliminação gradual e inclusive a erradicação da doença parecia estar ao alcance. Contudo, acrescentamos, contrariando este otimismo, presenciamos a situação presente.

Dentro desta mesma linha de raciocínio, David Tejada de Rivero ${ }^{10}$ (ex-subdiretor geral da OMS e coordenador geral da Conferência Internacional sobre Atenção Primária de Saúde/Alma-Ata) faz uma reflexão sobre a frustração e a dívida social para o século XXI. O autor mostra o que é, e o que não é, Atenção Primária. A meta de saúde para todos no ano 2000 deixou de ser cumprida: os governos confundiram metas sócio-políticas com metas programáticas, havendo assim múltiplas distorções. Os governos usaram equivocadamente cifras médias nacionais e informações questionáveis, e não tomaram consciência do fato de que nunca houve na História tantas e tão profundas diferenças e injustiças sociais que aumentam dia a dia.

\section{REFERÊNCIAS BIBLIOGRÁFICAS}

1. Fundação Nacional de Saúde. Plano Nacional de Controle da Tuberculose. Ministério da Saúde, Brasília,1999.

2. Jones LR, Thompson F. Um modelo para nova gerência pública. Revista do Serviço Público 51:41-79, 2000.

3. Ministério da Saúde. Manual de Administração/ Programa Nacional de Controle da Tuberculose. Boletim de Pneumologia Sanitária 4:7-56, 1996.

4. Pio A. El futuro de la lucha antituberculosa. Problemas y perspectives. Boletin de la Oficina Sanitaria Panamericana 96:108-118, 1984.

5. Rocha ATS. Medidas para o controle da tuberculose na comunidade. In Situação e Perspectivas do controle das doenças infecciosas e parasitárias. Cadernos da Universidade Nacional de Brasília 3:127-132, 1981.

6. Ruffino-Netto A. Brasil: doenças emergentes ou reemergentes? Medicina, Ribeirão Preto 30:405, 1997.

7. Ruffino-Netto A. Impacto da reforma do setor saúde sobre os serviços de tuberculose no Brasil. Boletim de Pneumologia Sanitária 7:7-18, 1999.

8. Ruffino-Netto A, Pereira JC. Mortalidade por tuberculose e condições de vida: o caso Rio de Janeiro. Revista Saúde em Debate 12: 27-34, 1981

9. Starfield B. Medicion de los logros de la Atencion Primaria. In: White KL (ed) Investigaciones sobre Servicios de Salud: uma 
antologia. OPAS, Washington DC,. Publicacion Cientifica $\mathrm{n}^{\circ} 534$, p. 761-769.1992.

10. Tejada-de-Rivero $D$. Atenção primária. Frustração e dívida social para o século XXI? Revista Ser Médico 6:12-15, 1999.

11. World Health Organization. Global Tuberculosis Control. WHO Report, WHO/TB/98.237, 1998.

12. World Health Organization. Global Tuberculosis Control. WHO/ CDS/TB/2000. 275 WHO Report 2000.
13. World Health Organization. What is DOTS? A guide to understanding the WHO-recommended Tb control strategy known as DOTS. WHO/cds/cpc/tb/99.270, 1999.

14. World Health Organization. Amsterdam Declaration to Stop Tb. The Ministerial Conference on Tuberculosis \& Sustainable Development. Amsterdam, The Netherlands, 24 March, 2000. 